BUTP-97/35

hep-ph/9711431

\title{
NOTE on the MASS SQUARE of the ETA' MESON
}

\author{
P. Minkowski \\ Institute for Theoretical Physics \\ University of Bern \\ CH - 3012 Bern, Switzerland
}

22. November 1997

\begin{abstract}
We propose to use as a mathematical tool to study the mass square of the eta' meson an infinitely heavy supplementary quark flavor $\mathrm{Q}$. This is understood in the framework of QCD with one light quark flavor ( $\rightarrow n_{f l}$ light flavors) and the gauge group $S U 3_{c}\left(\rightarrow S U N_{c}\right)$. The full system thus consists of $n_{f l}^{(+)}=n_{f l}+1$ flavors, including the infinitely heavy one. The purpose of this note is to show how the heavy flavor can be made to represent the anomalies of the subtheory describing the remnant physical degrees of freedom. In the large $N_{c}$ limit the mass square of eta' tends to a finite limit. The essential deviations from (semi) perturbative derivations are related to simple properties of the 'heavy' flavor.
\end{abstract}

\footnotetext{
${ }^{1}$ Work supported in part by the Schweizerischer Nationalfonds.
} 


\section{Central anomalies, single local operator insertions}

We first turn to the trace anomaly [1] to demonstrate the method of using a limiting 'heavy' auxiliary flavor of quark and antiquark.

All quantities which include the auxiliary flavor shall be marked with the superfix ${ }^{(+)}$. The operator identity for the trace of the energy momentum tensor with

$$
n_{f l}^{(+)}=n_{f l}+1 ; n_{f l} \geq 1
$$

then takes the form

$$
\begin{aligned}
& \left(\vartheta^{(+)}\right)_{\mu}^{\mu}=-b_{1}^{(+)} \mathcal{B}^{2} \frac{1}{8 \pi^{2}}+m_{q} \bar{q} q+m_{Q} \bar{Q} Q \\
& \mathcal{B}^{2}=\mathcal{B}^{2}\left(N_{c}\right)=\left[\frac{1}{4} F_{\mu \nu}^{a} F^{a \mu \nu}\right] ; \quad b_{1}^{(+)}=\frac{11}{3} N_{c}-\frac{2}{3} n_{f l}^{(+)}
\end{aligned}
$$

All operators in eq. (2) are local and renormalization group invariant with vanishing anomalous dimension. In particular the field strengths forming the bilinear composite operator $\mathcal{B}^{2}$ include the coupling constant as multiplicative factor, relative to their perturbative variants. For details of the precise definitions we refer to [1] and [2]. Furthermore $\mathcal{B}^{2}$ does not depend on the number of quark flavors. $-b_{1}^{(+)}$stands for the first coefficient of the (relative) Callan-Symanzik function $\beta^{(+)}(g) 16 \pi^{2} / g^{3}$.

Appropriate sums over flavor and color are understood in the definition of the light flavor mass term $m_{q} \bar{q} q$. The flavor basis including the 'heavy' $\mathrm{Q}$ is chosen such, that the (scheme dependent) quark masses are all nonnegative. The 'heavy' flavor limit corresponds to $m_{Q} \rightarrow \infty$.

In parallel with the operator $(\vartheta(+))_{\mu}^{\mu}$ we consider its counterpart $(\vartheta)_{\mu}^{\mu}$, excluding from consideration the 'heavy' quark flavor. The corresponding trace identity then reads :

$$
\begin{aligned}
& (\vartheta)_{\mu}^{\mu}=-b_{1} \mathcal{B}^{2} \frac{1}{8 \pi^{2}}+m_{q} \bar{q} q ; b_{1}=\frac{11}{3} N_{c}-\frac{2}{3} n_{f l} \\
& (\Delta \vartheta)_{\mu}^{\mu}=(\vartheta(+))_{\mu}^{\mu}-(\vartheta)_{\mu}^{\mu}=\frac{\mathcal{B}^{2}}{12 \pi^{2}}+m_{Q} \bar{Q} Q
\end{aligned}
$$


The key point involves the vanishing of the operator $(\Delta \vartheta)_{\mu}^{\mu}$ defined in eq. (3), when inserted into Green functions pertinent to the subtheory with only $n$ fl flavors of quark and appropriately restricted momenta (or distances) in the 'heavy' flavor limit $m_{Q} \rightarrow \infty$.

We illustrate this considering the two gluon matrix element to lowest nontrivial order

$$
\begin{aligned}
& \left\langle\varepsilon_{2}, a_{2}, k_{2}\left|(\Delta \vartheta)_{\mu}^{\mu}\right| \varepsilon_{1}, a_{1}, k_{1}\right\rangle= \\
& \delta_{a_{2} a_{1}} \varepsilon_{2}^{* \sigma_{2}} \varepsilon_{1}^{\sigma_{1}^{1}}\left[g_{\sigma_{2} \sigma_{1}}\left(k_{2} k_{1}\right)-\left(k_{1}\right) \sigma_{2}\left(k_{2}\right)_{\sigma_{1}}\right] \Pi+\ldots \\
& \Pi=\Pi\left(k_{1}^{2}, k_{1}^{2}, k_{3}^{2}\right) \sim \frac{g^{2}}{12 \pi^{2}}+\Pi\left[m_{Q} \bar{Q} Q\right]
\end{aligned}
$$

In eq. (41) the dots represent all remaining covariant tensors ( $\left.O k^{4}\right)$ compatible with color gauge invariance. $k_{3}=k_{2}-k_{1}$ is the momentum transfer.

$\Pi\left[m_{Q} \bar{Q} Q\right]$ denotes the contribution of the 'heavy' quark insertion alone. The latter is readily calculated to the given order :

$$
\begin{aligned}
& \Pi\left[m_{Q} \bar{Q} Q\right]=\frac{g^{2}}{8 \pi^{2}} \Pi_{1}^{(Q)} \\
& \Pi_{1}^{(Q)}=\int d \Omega_{3}\left(4 \beta_{1} \beta_{2}-1\right) \times \\
& \times\left[\frac{m_{Q}^{2}}{X\left(k_{1}^{2}, k_{2}^{2}, k_{3}^{2}\right)}+\frac{m_{Q}^{2}}{X\left(k_{2}^{2}, k_{1}^{2}, k_{3}^{2}\right)}\right] \\
& X\left(k_{1}^{2}, k_{2}^{2}, k_{3}^{2}\right)=m_{Q}^{2}-\left[\beta_{2} \beta_{3} k_{1}^{2}+\beta_{1} \beta_{3} k_{2}^{2}+\beta_{1} \beta_{2} k_{3}^{2}\right] \\
& =\Omega_{3}=\delta_{\left(1-\sum_{k}^{3} \beta_{k}\right) \prod_{n}^{3} \vartheta\left(\beta_{n}\right) d \beta_{n}}
\end{aligned}
$$

In the 'heavy' flavor limit the expression in eq. (5) becomes 


$$
\begin{aligned}
& \lim _{m_{Q} \rightarrow \infty \Pi\left[m_{Q} \bar{Q} Q\right]}=-\frac{g^{2}}{12 \pi^{2}} \rightarrow \lim _{m_{Q} \rightarrow \infty} \Pi=0 \\
& 2 \int d \Omega_{3}\left(4 \beta_{1} \beta_{2}-1\right)=-\frac{2}{3}
\end{aligned}
$$

The quark part of the trace anomaly is revealed through the nontrivial 'heavy' mass insertion $\left[m_{Q} \bar{Q} Q\right]$ in eq. (6).

In a similar way we discuss the axial current anomaly, considering the two axial currents

$$
\begin{aligned}
& a_{\mu}^{(+)}=\bar{q} \gamma_{\mu} \gamma_{5} q-n_{f l} \bar{Q} \gamma_{\mu} \gamma_{5} Q ; a_{\mu}=\bar{q} \gamma_{\mu} \gamma_{5} q \\
& \gamma_{5}=\gamma_{5} R=-i \gamma_{0} \gamma_{1} \gamma_{2} \gamma_{3}
\end{aligned}
$$

From the two currents defined in eq. (7) we form the divergences

$$
\begin{aligned}
& D^{(+)}=\partial^{\mu} a_{\mu}^{(+)}=2 m_{q} \bar{q} i \gamma_{5} q-2 n_{f l} m_{Q} \bar{Q} i \gamma_{5} Q \\
& D=\partial^{\mu} a_{\mu}=2 m_{q} \bar{q} i \gamma_{5} q+2 n_{f l} \mathcal{B} \widetilde{\mathcal{B}} \frac{1}{8 \pi^{2}} \\
& \mathcal{B} \widetilde{\mathcal{B}}=\mathcal{B} \widetilde{\mathcal{B}}\left(N_{c}\right)=\left[\frac{1}{4} F_{\mu \nu}^{a} \widetilde{F}^{a \mu \nu}\right] ; \widetilde{F}_{\mu \nu}^{a}=\frac{1}{2} \varepsilon_{\mu \nu \sigma \tau} F^{a \sigma \tau}
\end{aligned}
$$

and their difference

$$
\begin{aligned}
& \Delta D=D^{(+)}-D=-2 n_{f l} \Delta^{(5)} \\
& \Delta^{(5)}=\mathcal{B} \widetilde{\mathcal{B}} \frac{1}{8 \pi^{2}}+m_{Q} \bar{Q} i \gamma_{5} Q
\end{aligned}
$$

As in eq. (田) we evaluate the two gluon matrix element of $\Delta^{(5)}$ in lowest order 


$$
\begin{aligned}
& \left\langle\varepsilon_{2}, a_{2}, k_{2}\left|\left(\Delta^{(5)} \vartheta\right)_{\mu}^{\mu}\right| \varepsilon_{1}, a_{1}, k_{1}\right\rangle= \\
& \delta_{a_{2} a_{1}} \varepsilon_{2}^{* \sigma_{2}} \varepsilon_{1}^{\sigma 1} \varepsilon_{\sigma_{2} \sigma_{1} \mu_{2} \mu_{1}}\left(k_{2}\right)^{\sigma_{2}}\left(k_{1}\right)^{\sigma_{1}} \Pi^{(5)}+\ldots \\
& \Pi^{(5)}=\Pi^{(5)}\left(k_{1}^{2}, k_{1}^{2}, k_{3}^{2}\right) \sim-\frac{g^{2}}{8 \pi^{2}}+\Pi^{(5)}\left[m_{Q} \bar{Q} i \gamma_{5} Q\right]
\end{aligned}
$$

The notation is analogous to the one in eq. (舟).

The quantity $\Pi{ }^{(5)}\left[m_{Q} \bar{Q} i \gamma_{5} Q\right]$ now relates to the pseudoscalar mass insertion corresponding to $m_{Q} \bar{Q} i \gamma_{5} Q$. The pseudoscalar mass insertion is readily evaluated [3], in line with the scalar mass insertion in eq. (5)

$$
\begin{aligned}
\Pi^{(5)}\left[m_{Q}\right. & \left.\bar{Q} i \gamma_{5} Q\right]=\frac{g^{2}}{8 \pi^{2}} \Pi_{1}^{(5)(Q)} \\
\Pi_{1}^{(5)(Q)} & =\int d \Omega_{3} \times \\
& \times\left[\frac{m_{Q}^{2}}{X\left(k_{1}^{2}, k_{2}^{2}, k_{3}^{2}\right)}+\frac{m_{Q}^{2}}{X\left(k_{2}^{2}, k_{1}^{2}, k_{3}^{2}\right)}\right]
\end{aligned}
$$

The quantities $d \Omega_{3}$ and $\mathrm{X}$ in eq. (11) are defined in eq. (5). Analogously to the scalar mass insertion in eq. (6) we find in the 'heavy' flavor limit

$$
\begin{aligned}
& \lim _{m_{Q} \rightarrow \infty} \Pi^{(5)}\left[m_{Q} \bar{Q} Q\right]=\frac{g^{2}}{8 \pi^{2}} \rightarrow \lim _{m_{Q \rightarrow \infty}} \Pi^{(5)}=0 \\
& 2 \int d \Omega_{3}=1
\end{aligned}
$$

This concludes the illustration how single local 'heavy' quark operators generate the quark induced parts of the central anomalies. This evidently exhausts the axial current anomaly. The trace anomaly associated with gauge boson self interaction - dominant for large $N_{c}$ - can only be represented by 'extended heavy' flavors, fermions and (pseudo)scalars, within the $N=4$ 
susy theory. The corresponding 'heavy' mass terms of course violate $N=4$ supersymmetry.

\section{Mass square of $\eta^{\prime}$, double local operator insertions}

To study the properties of $\eta^{\prime}$, we use the axial current $a_{\mu}^{(+)}$defined in eq. (7) and its divergence $D^{(+)}=\partial^{\mu} a_{\mu}^{(+)}$given in eq. (8). In particular we consider the correlation function of two divergencies

$$
\chi^{(+)}\left(q^{2}\right)=i \int d^{4} x e^{i q x}\left\langle\Omega\left|T\left[D^{(+)}(x) D^{(+)}(0)\right]\right| \Omega\right\rangle
$$

In the following we set all light quark masses to zero, when relevant. The $S U n_{f l}$ singlet pseudoscalar meson contributes, eventually with a finite width to $\chi^{(+)}$which is of the general form

$$
\chi^{(+)}\left(q^{2}\right)=2 n_{f l} \frac{(f(+))_{\eta^{\prime}}^{2}\left(q^{2}\right) m_{\eta^{\prime}}^{4}\left(q^{2}\right)}{m_{\eta^{\prime}}^{2}\left(q^{2}\right)-q^{2}}
$$

In eq. (14) the quantities $m_{\eta}^{2},\left(q^{2}\right)$ and $\left.f(+)\right)_{\eta}^{2},\left(q^{2}\right)$ are extended to arbitrary values of $q^{2}$, as off shell mass and decay constant (square) of $\eta^{\prime}$. In the form given above, eq. (14) is exact for all values of $q^{2}$. The Ward identity for the axial current $a_{\mu}^{(+)}$in eq. (8) implies

$$
\begin{aligned}
& \chi^{(+)}\left(q^{2}\right)= \\
& \left\{\begin{array}{c}
q^{\mu} \int d^{4} x e^{i q x}\left\langle\Omega\left|T\left[a_{\mu}^{(+)}(x) D^{(+)}(0)\right]\right| \Omega\right\rangle \\
-i \int d^{4} x e^{i q x} \delta(t)\left\langle\Omega\left|\left[a_{0}^{(+)}(x), D^{(+)}(0)\right]\right| \Omega\right\rangle
\end{array}\right\}
\end{aligned}
$$

The equal time commutator in eq. (15) involves the 'heavy' flavor only. It gives rise to the induced contact term, which I have discussed earlier [4], yielding a remnant contribution, persisting when we evaluate $\chi^{(+)}$at zero momentum :

$$
\chi^{(+)}(0)=4 n_{f l}^{2}\left\langle\Omega\left|\left(-m_{Q} \bar{Q} Q\right)\right| \Omega\right\rangle
$$


In the 'heavy' flavor limit it follows, combining eqs. (3) , (14) and (16)

$$
\begin{aligned}
& f_{\eta^{\prime}}^{2},(0) m_{\eta^{\prime}}^{2}(0)=\frac{n_{f l}}{3} \frac{1}{2 \pi^{2}}\left\langle\Omega\left|\mathcal{B}^{2}\right| \Omega\right\rangle \\
& f_{\eta^{\prime}}^{2}\left(q^{2}\right)=\lim _{m_{Q} \rightarrow \infty}(f(+)){ }_{\eta^{\prime}}^{2}\left(q^{2}\right)
\end{aligned}
$$

The relation in eq. (17) reveales the 'heavy' quark contact term as an anomalous contribution involving the insertion of two local operators. This affects the topological susceptibility through nontrivial boundary conditions

$$
\begin{aligned}
& \lim _{m_{Q} \rightarrow \infty} \chi{ }^{(+)}(0)= \\
& 4 n_{f l}^{2} i \int d^{4} x\left\langle\Omega\left|T\left[\operatorname{ch}_{2}(x) \operatorname{ch}_{2}(0)\right]\right| \Omega\right\rangle \\
& \operatorname{ch}{ }_{2}=\mathcal{B} \tilde{\mathcal{B}} \frac{1}{8 \pi^{2}}
\end{aligned}
$$

Eq. (17) falsifies the pertubatively motivated hypothesis [5] 2

$$
m_{\eta}^{2}, \propto 1 / N_{c}
$$

in the large $N_{c}$ limit, since both the gauge boson condensate $\left\langle\Omega\left|\mathcal{B}^{2}\right| \Omega\right\rangle$ and the square of the $\eta^{\prime}$ decay constant $f_{\eta}^{2},(0)$ are proportional to $N_{c}$ in that limit.

Thus the extrapolation in $q^{2}$ from $m_{\eta}^{2}$, to zero does not allow a straightforward identification of $m_{\eta^{\prime}}(0)$ with the physical mass of $\eta^{\prime}$ nor of $f_{\eta^{\prime}}(0)$ with its decay constant.

We rescale the remnant relations combining eqs. (16) and (18) in the 'heavy' flavor limit to the topological susceptibility per unit winding number $\chi_{c h}$

$$
\begin{gathered}
\lim _{m_{Q} \rightarrow \infty} \chi^{(+)}(0)=4 n_{f l}^{2} \chi_{c h} ; \Theta_{F}=\mathcal{B}^{2} \frac{1}{12 \pi^{2}} \\
\chi_{c h}=i \int d^{4} x\left\langle\Omega\left|T\left[\operatorname{ch}_{2}(x) \operatorname{ch}_{2}(0)\right]\right| \Omega\right\rangle=\left\langle\Omega\left|\Theta_{F}\right| \Omega\right\rangle
\end{gathered}
$$

\footnotetext{
${ }^{2}$ We refrain from citing here any of the numerous more recent papers, expanding on the incorrect large $N_{c}$ limit.
} 
$\chi_{c h}$ defined in eq. (20) is to be evaluated retaining all light flavors of gauge bosons and quarks.

The Euclidean space version of eq. (20) in a finite large fourdimensional volume $V=V_{4}$ becomes using thermodynamic notation [6] $\chi_{c h} \rightarrow$ $(\Delta \nu)^{2}(V) / V$

$$
\begin{aligned}
& (\Delta \nu)^{2}(V)=N_{F}(V) \rightarrow \varrho_{F} V \\
& \varrho_{F}=\left\langle\Omega\left|\Theta_{F}\right| \Omega\right\rangle \propto N_{c}
\end{aligned}
$$

There is a definite difference in sign - not an error - in the definition of the quantities $\chi_{c h}$ and $\Delta \nu^{2}$ in eqs. (20) and (21) relative to ref. [6] . Which correlation function is positive is controlled here by the 'heavy' flavor and the sign of the gauge boson condensate. The sign in question reveales an interesting property of Euclidean relative time correlations with respect to their physical time counterparts.

We conclude by evaluating, despite the large extrapolation, the relation in eq. (17) using the value of the gauge boson condensate derived by Shifman, Vainshtain and Zakharov from charmonium sum rules [7], the pion decay constant for $f_{\eta^{\prime}}(0)$ and the physical mass of $\eta^{\prime}$ for $m_{\eta^{\prime}}(0)$

$$
\begin{aligned}
& \left\langle\Omega\left|\mathcal{B}^{2}\right| \Omega\right\rangle \sim 0.125 \mathrm{GeV}^{4} \\
& f_{\eta^{\prime}}(0) \sim f_{\pi}=0.0932 \mathrm{GeV}
\end{aligned}
$$

We find

$$
m_{\eta}^{2}, \sim \frac{n_{f l}}{3} 0.73 \mathrm{GeV}^{2} ; m_{\eta}^{2},-\frac{2}{3} m_{K}^{2}=0.75 \mathrm{GeV}^{2}
$$

For comparison we reduced the physical value of $m{ }_{\eta}^{2}$, by $\frac{2}{3} m{ }_{K}^{2}$ to offset the nonvanishing mass of the strange quark, ignoring $\eta \eta^{\prime}$ mixing. Taking the latter into account improves the numerical agreement beyond the theoretical error in the gauge boson condensate.

\section{Acknowledgments}

I should like to thank C, Greub and M. Leibundgut for raising pertinent questions and for the ensuing discussions. 


\section{References}

[1] P. Minkowski, Bern University preprint (1976), N. K. Nielsen, Nucl. Phys. B120 (1977) 212 ,

S.L. Adler, J.C. Collins and A. Duncan, Phys. Rev. D15 (1977) 1712 ,

J.C. Collins, A. Duncan and S.D. Joglekar, Phys. Rev. D16 (1977) 438.

[2] M. Leibundgut and P. Minkowski, Bern University preprint (1997) , BUTP-97/22, hep-th/9708061.

[3] S. Adler, Lectures in Elementary Particles and Quantum Field Theory, S. Deser, M. Grisaru and H. Pendleton edts., MIT Press, Cambridge Mass. 1970, 1970 Brandeis University Summer Institute, Vol. 1, 1-164.

[4] P. Minkowski, Phys. Lett. B237 (1990) 531.

[5] E. Witten, Nucl. Phys. B156 (1979) 269, G. Veneziano, Nucl. Phys. B159 (1979) 213.

[6] R. J. Crewther, Riv. Nuovo Cim. 2 (1979) 63, G. 't Hooft Phys. Rev. D14 (1976) 3432, (E) Phys. Rev. D18 (1978) 2199.

[7] M.A. Shifman, A.I. Vainshtain and V.I. Zakharov, Nucl. Phys. B147 (1978) 385, 448. 